\title{
Anabases
}

ANABASES Traditions et réceptions de l'Antiquité

$23 \mid 2016$

Varia

\section{François HARTOG, Partir pour la Grèce}

\section{Pascal Payen}

\section{OpenEdition \\ Journals}

Édition électronique

URL : http://journals.openedition.org/anabases/5699

DOI : 10.4000/anabases.5699

ISSN : 2256-9421

\section{Éditeur}

E.R.A.S.M.E.

\section{Édition imprimée}

Date de publication : 2 mai 2016

Pagination : 318-319

ISSN : 1774-4296

\section{Référence électronique}

Pascal Payen, « François HaRtog, Partir pour la Grèce », Anabases [En ligne], 23 | 2016, mis en ligne le 02 mai 2016, consulté le 22 septembre 2020. URL : http://journals.openedition.org/anabases/5699 ; DOI : https://doi.org/10.4000/anabases.5699

Ce document a été généré automatiquement le 22 septembre 2020.

(c) Anabases 


\title{
François HARTOG, Partir pour la Grèce
}

\author{
Pascal Payen
}

\section{RÉFÉRENCE}

François HARTOG, Partir pour la Grèce

Paris, Flammarion, 2015, 286 p.

23,90 euros/ ISBN 978-2-0813-3797-8

1 Depuis plusieurs décennies, François Hartog constitue une œuvre qui s'organise autour de trois composantes. La première réunit des livres (Le miroir d'Hérodote. Essai sur la représentation de l'autre, 1980 ; Mémoire d'Ulysse. Récits sur la frontière en Grèce ancienne, 1996) qui relèvent d'une histoire culturelle du monde antique, autour des problèmes de l'altérité et de la frontière. Une autre composante rassemble des recherches portant sur l'écriture de l'histoire, ancienne et moderne (L'Histoire, d'Homère à Augustin, 1999 ; Évidence de l'histoire. Ce que voient les historiens, 2005). Une troisième orientation concerne les usages modernes de l'Antiquité (Régimes d'historicité, 2003 ; Croire en l'histoire, 2013). Dans les trois formes de réflexion, c'est le problème du rapport au temps, plus encore l'analyse des " allers et retours entre des temps différents ", qui se présente comme " une contribution à une histoire [...] intellectuelle de la culture européenne »(Anciens, Modernes, Sauvages, 2005, p. 20). Le livre qui paraît, Partir pour la Grèce, condense dans son titre les trois approches, en se présentant comme "une invitation à s'interroger sur notre rapport à la Grèce ". Si, répondant à l'invitation, on entreprend le voyage, on doit tout d'abord constater que la Grèce n'appartient plus tout à fait aux Grecs depuis longtemps. Au moins depuis que " les Romains, les premiers, pour répondre à l'inévitable question "Qui sommes-nous ?", ont dû se confronter aux Grecs » (p. 10). À moins que l'on ne prenne un repère entre le $\mathrm{IV}^{\mathrm{e}}$ et le $\mathrm{VI}^{\mathrm{e}}$ siècle après J.C., marquant l'effacement de l'empreinte culturelle de la civilisation grecque, tel que la fermeture, par l'empereur Justinien, de l'école platonicienne d'Athènes, en 529. L'histoire de ces renoncements, de ces oublis pourrait être reconstituée et l'a déjà été en partie. Fort différemment, mais aussi avec cette histoire comme horizon lointain et 
nécessaire à la fois, le projet de ce livre est de s'intéresser à ceux qui ont décidé de nouer de nouveaux liens avec cet héritage et donc de " partir pour la Grèce ». Quelle nécessité les a poussés ? Qu'en ont-ils retiré ? Quelle nouvelle, quelle autre Grèce ancienne ont-ils vue?

Les six chapitres du livre sont entourés d'une longue préface, « La Grèce vient de loin ", ainsi que d'une ouverture et d'un épilogue. Toutes ces sections ont pour fonction de délimiter et de décrire les différentes modalités du « départ », ou plutôt quelques-unes d'entre elles, selon que l'on privilégie telle figure de voyageur (Moses Finley, Paul Ricœur, d'autres encore) ou tel lieu marquant le départ, avec ses héritages culturels, comme les chapitres 5 et 6 consacrés à " Une Grèce à la française ». Le livre ne se présente pas comme une histoire en continu de ces moments, de ces voyageurs en partance pour la Grèce, de ces regards, mais reconstitue des étapes clés, avec toujours cette question : comment, à partir du présent, à partir des différents présents, a-t-on esquissé, puis formalisé un mouvement de retour vers les Grecs ? Pourquoi continue-ton, maintenant, de le faire, alors même que le contexte ne semble pas s'y prêter (ainsi de la récente mise à l'écart, mise au placard des langues et civilisations anciennes dans les programmes des collèges, et de la probable exclusion de l'histoire grecque, du programme du CAPES, pour les sessions de 2017 et 2018 [voir ici même, p. 185-216]) ? Ce livre ne porte pas non plus sur l'expérience des récits de voyage en Grèce, sous une forme savante ou, plus récemment, touristique. Il n'est pas non plus question de " retours ", lourds d'un dépaysement. Tout autre, " la Grèce visée est une Grèce vue d'ailleurs »; "[elle] est appréhendée comme construction, comme représentation, comme objet de savoir, de désir aussi et de nostalgie ", " une Grèce prise au second degré » ; " c'est au fond cette Grèce-là qui m’a toujours le plus intéressé ", écrit François Hartog (p. 45-46), Mais cet héritage n'est pas le fait d'une expérience solitaire. La traversée s'est toujours faite en compagnie de quelques grands passeurs, dont les noms reviennent régulièrement, rythmant d'autant d'étapes le voyage : Fustel de Coulanges, Gernet, Momigliano, Finley, Vidal-Naquet, Vernant, et d'autres tels que Winckelmann. Avec lui, il s'agit bien non pas de copier les Grecs, mais de puiser directement à la source grecque, pour se construire soi-même. Ces départs pour la Grèce posent ainsi le problème de l'imitation. À deux reprises est citée une lettre écrite par Guillaume de Humboldt, à Goethe, depuis Rome, en 1804: " Ce serait une illusion que de souhaiter être les citoyens d'Athènes ou de Rome. C'est seulement dans la distance, dans la séparation d'avec l'ordinaire, seulement comme passé révolu que doit nous apparaître l'Antiquité » (p.60 et 143). Celle-ci participe d'un double processus d'idéalisation et d'historicisation. Dans cette fabrique de l'Antiquité intervient encore une série de couples " qui ont structuré dans la longue durée les manières dont l'Europe en est venue à se représenter elle-même [par le recours aux Grecs] »: successivement Grecs/Barbares, Chrétiens/Païens, Anciens/Modernes, " couples dynamiques, asymétriques, antagonistes et inséparables [qui] ne se sont jamais stabilisés " (p.43), et qui illustrent aussi comment se mettent en place, en Europe, particulièrement aux $\mathrm{XVIII}^{\mathrm{e}}$ et $\mathrm{XIX}^{\mathrm{e}}$ siècles, des rapports profondément divergents à la Grèce, au milieu desquels émerge la complexité du " départ allemand ».

Tout en analysant les singularités de ces départs pour la Grèce, François Hartog pose la question de savoir s'ils ont un point commun et privilégie la réponse que suggère Vernant : l'expérience grecque est avant tout politique et elle se trouve condensée dans ce qui caractérise la polis comme lieu du débat public, dont les règles elles-mêmes 
constituent les premiers éléments à être l'objet de la discussion. La cité tient aussi dans ses marges, que la démocratie politique laisse à l'écart, mais qui représentent autant de points de vue sur elle : les femmes, les jeunes, les esclaves, les étrangers, Grecs ou non, groupes sociaux que l'historiographie et l'anthropologie réintègrent dans le cadre civique et, à bonne place, dans le champ des sciences humaines et sociales.

4 Est-ce suffisant pour préserver - et pour combien de temps - les études grecques et latines, alors que le statut de ces langues sera bientôt celui qui est réservé actuellement à l'akkadien ou au hittite ? Mieux vaut s'en tenir au constat lucide que formule François Hartog : depuis quarante ans, "globalement, la culture classique achève de disparaître " (p. 225). Dès lors l'Antiquité, enfin réunie, mais à quel prix, pourrait représenter ce qu'Yves Bonnefoy appelle un "arrière-pays ", un fond commun omniprésent de valeurs sociales et politiques. À chacun d'en décider et de s'engager pour qu'il en soit ainsi. C'est « l'armature intellectuelle de l'Europe » qui est en jeu, et « ce qui, encore une fois, [1']a rendu[e] possible » (p. 43).

\section{AUTEURS}

\section{PASCAL PAYEN}

Université Toulouse-Jean Jaurès (UT2J)

payen@univ-tlse2.fr 